\title{
MULTIPLE PITCH IDENTIFICATION USING COCHLEAR-LIKE FREQUENCY CAPTURE AND HARMONIC GROUPING
}

\author{
Ramdas Kumaresan, Vijay Kumar Peddinti \\ Department of Electrical Engineering, Kelley Hall
University of Rhode Island
Kingston, RI 02881
}

\author{
Peter Cariani \\ Department of Otology \& Laryngology, \\ Harvard Medical School \\ Boston, MA 02114
}

\begin{abstract}
This work addresses the problem of identifying multiple fundamental frequencies in an acoustic signal. An auditoryinspired peripheral signal processing model is proposed that functions in a manner more like a bank of FM receivers rather than a traditional filterbank. Such receivers lock on to a strong signal (synchrony capture, frequency capture) even in the presence of nearby only slightly weaker signal components. Once the individual signal components are resolved, the model subjects them to an instantaneous nonlinearity and then performs harmonic grouping by cross correlating the isolated components. After the harmonically-related components are grouped, their pitches are computed using a standard summary autocorrelation approach.
\end{abstract}

Index Terms - Pitch, Harmonics, Cochlea

\section{INTRODUCTION}

Pitch is an essential attribute of periodic acoustic signals in speech, music, and other listening contexts. For a periodic sound, pitch is almost invariably heard at its fundamental frequency $f_{0}$. Common onset and common periodicity ("harmonicity") are the two strongest factors in grouping frequency components into auditory objects, and separating out multiple objects, each with its own pitch. Neural pitch mechanisms thus appear to be intimately related to early auditory grouping mechanisms, which in turn render analyses of multiple objects and streams in the auditory scene much more tractable. Human listeners are presently far superior to artificial, machine listening systems when it comes to tracking and analyzing sounds in noisy, cluttered, real world acoustic environments. If the operating principles inherent in neural mechanisms for pitch and auditory grouping can be understood, better artificial speech and music recognition systems and auditory prostheses are likely to follow. With this in mind a method for identifying pitches of multiple sets of harmonic

This work was supported by the Airforce Office of Scientific Research under the grant \# AFSOR FA9550-09-1-0119 sounds is proposed that may have parallels with signal processing strategies that are employed by 1 auditory systems.

Three major classes of pitch models exist: spectral pattern-matching models, residue models, and temporal autocorrelation models [1]. Spectral pattern-matching mechanisms analyze patterns of resolved frequency components and group harmonically-related components into separate auditory objects (via template matching [2], neural nets, or subharmonic superpositions). Residue models rely on interactions of unresolved harmonics (beating) produced by broad cochlear filtering and carry out a temporal analysis of the resultant periodicities. Temporal autocorrelation and cancellation models analyze patterns of all-order interspike intervals produced in the auditory nerve to identify interval peaks $[3,4]$ or subpatterns associated with different pitches.

Spectral pattern theories depend entirely on resolved harmonics, and therefore cannot explain the somewhat weaker fundamental pitches produced by unresolved harmonics. Residue theories depend entirely on waveform interactions between unresolved harmonics, and therefore do not explain the stronger pitches produced by resolved harmonics. Temporal autocorrelation models operate on interspike intervals produced by both resolved and unresolved harmonics and pure tones, and therefore provide a unified account of pitches associated with periodicities below $4-5 \mathrm{kHz}$. [3].

Psychophysical research [5] indicates that separations of multiple auditory objects with different fundamentals crucially depend on the presence of resolved, non-interfering low harmonics. This implies some neural mechanism that depends on the separation of harmonics prior to the operation of harmonic grouping and formation of auditory objects.

Temporal discharge patterns in the auditory nerve provide a neurally- and psychophysically-plausible basis for central neural representations of frequency and periodicity, be they in either the frequency- [2] or time-domain [3]. Here the precise nature of temporal representations of resolved and unresolved harmonics can shed light on the neural mechanisms underlying separation of multiple concurrent harmonic sounds. If one examines the representation of low harmonics of complex 
sounds in the auditory nerve, a striking feature is "synchrony capture", wherein nerve fibers in an entire cochlear region are driven almost exclusively by one local frequency component [6], such that the individual component imposes its temporal fine structure on the temporal patterning of spikes in that region. Synchrony capture occurs at moderate and high sound pressure levels, where auditory nerve fibers are typically driven at their maximal rates over a range of pure tone frequencies of an octave or more. For low or resolved harmonics, synchrony capture enhances the interspike interval representations of the individual harmonics in fibers whose characteristic frequencies (CF's) are nearby. When harmonics are close together in frequency, within a critical band or so, surrounding auditory nerve $\mathrm{CF}$ regions are instead driven by the composite waveform pattern of the two interacting harmonics, and the interspike interval representation of individual harmonics is degraded. Thus, neural synchrony capture appears to parallel perceptual harmonic resolution. Because resolved harmonics permit separation of concurrent sounds, synchrony capture in artificial systems may likewise be exploited for better sound separations.

\section{SYNCHRONY/FREQUENCY CAPTURE MODEL}

With this in view, a model is proposed for peripheral processing that uses a snchrony capture mechanism to effect the capture of individual frequency components. Rather than a simple filter bank, the mechanism behaves more like a bank of FM receivers, and hence tends to capture the strong frequency components in a signal and mask weaker ones. Such receivers are known to lock on (or frequency capture [7]) to a strong FM signal even in the presence of nearby, only slightly weaker signals. After individual components are separated via frequency capture, a harmonic grouping operation is performed by a Harmonic Relation Detector (HARD) (block in Figure 1). Here the channel outputs are subjected to a nonlinear distortion, mutually cross-correlated, and then tested to determine if they are harmonically-related.

In the following, a simple adaptive signal processing approach is proposed that exhibits this type of frequency/synchrony capture behavior. The proposed implementation is motivated by the anatomy and biophysics of the cochlea, particularly the crystalline structure of the three rows of outer hair cells (OHCs) that ride on top of the basilar membrane. Motivated by these observations, an adaptive filter structure consisting of three bandpass filters (BPF) was envisioned, provisionally called a BPF triplet, that can home in on a dominant tone in an input signal. This idea was then used to synthesize a composite Synchrony Capture Filterbank (SCFB) that can be used as a front-end for further signal analysis. The BPF triplet structure is essentially the same as used by Costas [8].

First, a simple model is formulated for the log-envelope and phase derivative of a signal consisting of two tones, which is useful in explaining the synchrony capture phenomenon. Using this model, how a tunable BPF triplet can be designed

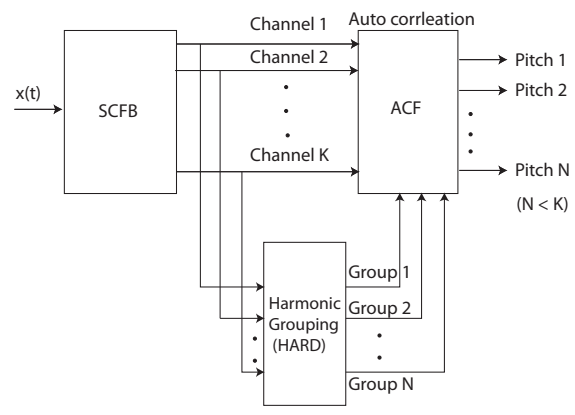

Fig. 1. Multiple pitch estimator: The SCFB (Synchrony Capture Filterbank) block consists of $\mathrm{K}$ peripheral filters, each of which is followed by a Bandpass filter Triplet shown in Figure 2. Each such cascade is called a channel. SCFB helps separate and capture the dominant frequency components in $x(t)$. HARD thus determines which harmonic components belong in a group, i.e. in a separate auditory object. The ACF unit computes the autocorrelation function for each channel output and obtains a pitch estimate for each group separately by computing the Summary ACFs (SACFs)[4]. K is the number of channels. $N$ is the number of harmonic groups. $K>>N$.

to follow a dominant tone in the input is outlined. Consider a signal $s(t)$ consisting of a tone at frequency $\omega_{1}\left(=2 \pi f_{1}\right)$ and an interfering tone at $\omega_{2}\left(=2 \pi f_{2}\right) . s(t)$ is a model for the output of one of the peripheral filters in the SCFB module.

$$
s(t)=A_{1} \cos \left(\omega_{1} t+\theta_{1}\right)+A_{2} \cos \left(\omega_{2} t+\theta_{2}\right)
$$

Let us assume that $A_{1}>A_{2}$. Then, it is easy to show that $s(t)$

$$
s(t) \approx a(t) \cos (\phi(t))
$$

where the envelope is

$$
a(t)=e^{\ln A_{1}+\frac{A_{2}}{A_{1}} \cos (\Delta \omega t+\Delta \theta)},
$$

and the phase function is

$$
\phi(t)=\omega_{1} t+\theta_{1}+\frac{A_{2}}{A_{1}} \sin (\Delta \omega t+\Delta \theta),
$$

and $\Delta \omega=\omega_{2}-\omega_{1}$ and $\Delta \theta=\theta_{2}-\theta_{1}$. The instantaneous frequency (IF) and the log-envelope are as follows.

$$
\begin{aligned}
& \frac{d \phi(t)}{d t}=\omega_{1}+\frac{A_{2}}{A_{1}} \Delta \omega \cos (\Delta \omega t+\Delta \theta), \\
& \ln a(t)=\ln A_{1}+\frac{A_{2}}{A_{1}} \cos (\Delta \omega t+\Delta \theta) .
\end{aligned}
$$

Note that the average value of IF is $\omega_{1}$, the dominant tone's frequency. A system can lock on to it by filtering the IF using a low-pass filter (LPF) with a cut off frequency $\Delta \omega$. This is the common frequency capture phenomenon [7] that occurs in traditional FM receivers such as FM discriminators and Phase 
Lock Loops (PLLs). Alternatively, envelope or amplitude information can also be used to capture the dominant signall. That is, the compressed envelope signal, $\ln a(t)$, can be low pass filtered to obtain $\ln A_{1}$. This can then be used to home in on the dominant tone in the input as outlined in Figure 2.
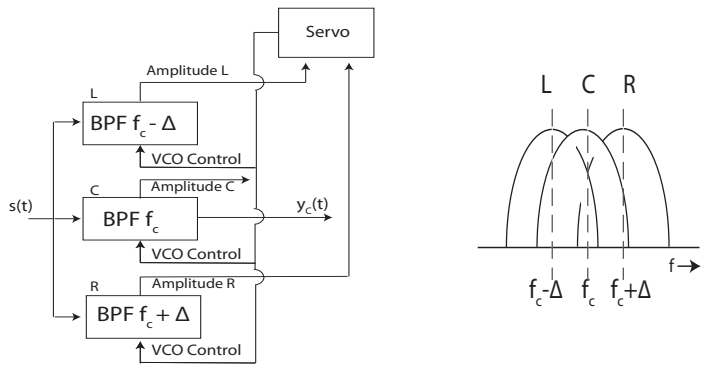

Fig. 2. Synchrony/Frequency Capturing BPF Triplet: $s(t)$ is the output of a peripheral filter in SCFB. Each peripheral filter inputs to a BPF triplet shown above. The left (L), Center (C) and Right (R) bandpass filters are centered at $f_{c}-\Delta, f_{c}$ and $f_{c}+\Delta$ $\mathrm{Hz}$ respectively. The center frequencies of the BPF triplets can be moved together by changing the frequencies of the VCOs which are used to tune them. The envelopes of the BPF filter outputs are filtered to get their slowly changing amplitudes. These are denoted by $\mathrm{L}, \mathrm{C}$ and $\mathrm{R}$. The quantity $\frac{R-L}{R+L}$ is used by the servo loop to change the frequencies of the VCOs. Thus, the filters are moved such that centre frequency $f_{c}$ of the center filter tracks the frequency of the dominant tone in $s(t)$. Track is achieved when $R=L$. The $\mathrm{C}$ filter output, $y_{c}(t)$, is the channel output.

\section{HARMONIC RELATION DETECTOR (HARD)}

Once the tones are isolated how do we determine if two tones $\cos \left(2 \pi f_{1} t+\theta_{1}\right)$ and $\cos \left(2 \pi f_{2} t+\theta_{2}\right)$ have a harmonic relationship, that is, they have a common fundamental $f_{0}$ ? Clearly, the range of $f_{0}$ must be restricted to avoid arbitrarily low $f_{0}$ values. Here $f_{0}$ has been restricted to the range of periodicity pitch, between 20 to $2000 \mathrm{~Hz}$. Therefore, we need to determine if $f_{1}$ and $f_{2}$ have a harmonic relationship over a maximum interval of $T_{i} \approx 50 \mathrm{~ms}(=1 / 20 \mathrm{~Hz}$.) Let us assume that $f_{1}$ and $f_{2}$ are indeed harmonically related, that is $f_{1}=n f_{0}$ and $f_{2}=p f_{0}$ where $n$ and $p$ are integers. We then subject the tones to an instantaneous nonlinearity such that they produce tones of all the higher integer multiples of $f_{1}$ and $f_{2}$. Chebyshev polynomials serve as a convenient nonlinear function [9]. That is, $g_{m}(t)$ is defined as the output of the nonlinearity (denoted by the NL box in Figure 3 ) when the input is a tone $\cos \left(2 \pi f_{m} t+\phi_{m}\right)$.

$$
g_{m}(t)=\sum_{k=1}^{N_{m}} T_{k}\left(\cos \left(2 \pi f_{m} t+\phi_{m}\right)\right), \quad m=1,2
$$

where $T_{k}$ are the Chebyshev polynomials. Notice that the nonlinear operation is such that it produces overtones with frequencies that are both odd and even multiples of $f_{1}$ (or $f_{2}$ ). Note also that the dc component is intentionally eliminated. Nonlinearities other than Chebyshev polynomials can also be used. The nonlinearly distorted tones are then mul-

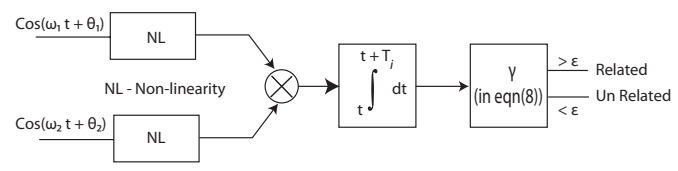

Fig. 3. Non-linear Interchannel Crosscorrelator: This unit is the building block for the harmonic relationship detector (HARD) in Figure 1. It produces the overtones of two sinusoids and computes their normalized crosscorrelation $\gamma$.

tiplied together and integrated over an interval of $T_{i}$ secs to compute the normalized cross correlation $\gamma$.

$$
\gamma=\left|\frac{\int g_{1}(t) g_{2}(t) d t}{\sqrt{\int g_{1}^{2}(t) d t \int g_{2}^{2}(t) d t}}\right|
$$

As an example, let $f_{1}$ and $f_{2}$ be 220 and $550 \mathrm{~Hz}$ respectively. $f_{1}=n f_{0}$ and $f_{2}=p f_{0}$ where $n=2, p=5$ and $f_{0}=110 \mathrm{~Hz}$. Then the two distorted tones $g_{1}(t)$ and $g_{2}(t)$ have common frequencies $1100,2200,3300$ etc which are integer multiples of $\operatorname{LCM}(\mathrm{n}, \mathrm{p}) \times f_{0}$, where LCM is the least common multiple. Thus $\gamma$ will tend to be large. If the frequencies are unrelated then $\gamma$ will be small.

\section{SIMULATION EXAMPLE}

In this paper, for lack of space, we restrict ourselves to applying the proposed multipitch identification algorithm to a synthetic signal $x(t)$ which consists of only two sets $(\mathrm{N}=2)$ of harmonics. The two fundamental frequencies are $f_{01}=109$ $\mathrm{Hz}$ and $f_{02}=123 \mathrm{~Hz}$.

$$
x(t)=\sum_{k=1}^{6} A_{k} \cos \left(2 \pi f_{k} t+\theta_{k}\right),
$$

where the frequencies $f_{1}$ to $f_{3}$ are 218,436 and $981 \mathrm{~Hz}$ ( integer multiples of $f_{01}$ ), while the frequencies $f_{4}$ to $f_{6}$ are 492 , 738 and $861 \mathrm{~Hz}$ ( integer multiples of $f_{02}$ ). $\theta_{k}$ are chosen randomly. All $A_{k}$ are unity. $x(t)$ is input to a gamma tone filter bank, the details of which are described elsewhere [10]. The filter bank consists of 50 filters spanning a frequency range of 100 to $1500 \mathrm{~Hz}$. The filters are relatively broad with $\mathrm{Q}=4$.

The output of each filter is fed to a BPF triplet shown in Figure 2. For each sinusoid in $x(t)$ with frequency $f_{k}$, the channels in that neighborhood stay locked on to it. Typically the channel with the center frequency closest to $f_{k}$ will have the maximum amplitude. For example, if we consider the case of $f_{1}=218 \mathrm{~Hz}$, the channel \#s 5 to 10 (with center frequencies ranging from $164 \mathrm{~Hz}$ and $253 \mathrm{~Hz}$ respectively) are locked on to $218 \mathrm{~Hz}$, and channel \#7 (whose center frequency is $223 \mathrm{~Hz}$ ) has the highest amplitude as it is closest to $218 \mathrm{~Hz}$. The outputs of those channels with maximum amplitude in a neighborhood are fed to the ACF calculator and the harmonic relation detector(HARD) in parallel. See Figure 1.

In the HARD block in Fig. 1 the channel outputs are nonlinearly distorted to produce some of the higher harmonics 
(see Eq. (7)). The nonlinearly distorted signals are then crosscorrelated to obtain the $\gamma$ values using formula in Eq.8. As an example, let us consider the spectra of distorted signals $g_{m}(t)$ in two different cases: (1) 218 and $981 \mathrm{~Hz}$, (2) 218 and 738 Hz. 218 and $981 \mathrm{~Hz}$ are harmonically related, where as 218 and $738 \mathrm{~Hz}$ are not (with in a time window of 50ms). Figure 4 shows the spectra of the corresponding distorted signals. Notice that the spectral lines coresponding to the higher harmonics of 218 and $981 \mathrm{~Hz}$ coincide at many locations (top panel) where as such coincidnces or near coincidences are less common in the case of 218 and $738 \mathrm{~Hz}$ (bottom panel). As a result the $\gamma$ for 218 and $981 \mathrm{~Hz}$ tones are higher than for 218 and $738 \mathrm{~Hz}$. Hence 218 and $981 \mathrm{~Hz}$ belong in group 1.

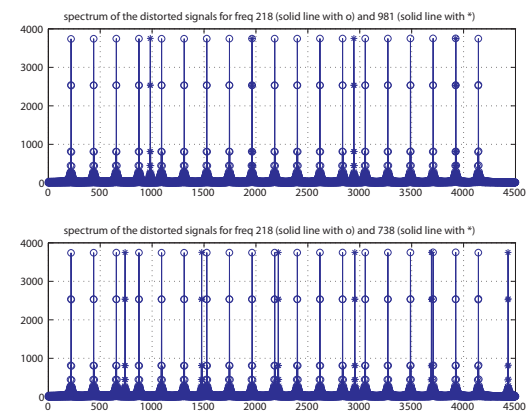

Fig. 4. Spectra of NL distorted signals corresponding to frequencies 218 and $981 \mathrm{~Hz}$ (top) and 218, $738 \mathrm{~Hz}$ (bottom). If the overtone frequencies are the same then the ' $\varnothing$ ' and '*'coincide. No exact coincidences are found in the bottom panel.

The 3D plot in Figure 5 displays the $\gamma$ values for all the 15 possible pairs of frequencies. Generally, if two frequencies are from the same group (either $f_{01}$ group or the $f_{02}$ group) then the corresponding $\gamma$ values tend to be larger. Note that harmonically related tones like $436 \mathrm{~Hz}$ and $981 \mathrm{~Hz}$ have large $\gamma$ values whereas $218 \mathrm{~Hz}$ and $738 \mathrm{~Hz}$ or 861 and $981 \mathrm{~Hz}$ have relatively small $\gamma \mathrm{s}$. Thus we can use these $\gamma$ values to group the tones that belong to a harmonic set. Once the tones are grouped, then the autocorrelation functions for the channel outputs for these groups can be summed. Figure 6 shows the Summary Autocorrelation for groups 1 and 2. The pitch periods corresponding to the two groups can then be determined by picking the first global peak away from lag zero.

\section{REFERENCES}

[1] A. de Cheveigne, "Pitch perception," in The Oxford Handbook of Auditory Science, Christopher J. Plack, Ed. Oxford, Oxford University Press Inc, New York.

[2] P. Srulovicz and J. Goldstein, "A central spectrum model: A synthesis of auditory nerve timing and place cues in monoaural communication offrequency spectrum," J Acoust Soc Am, vol. 73, pp. 1266-1276, 1983.

[3] P. A. Cariani and B. Delgutte, "Neural correlates of the pitch of complex tones. I. pitch and pitch salience. II.

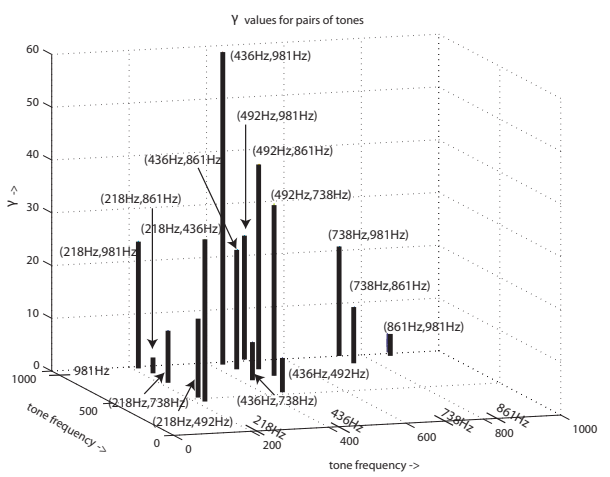

Fig. 5. $\gamma$ as a function of frequencies of pairs of tones. Note that harmonically related tones like $436 \mathrm{~Hz}$ and $981 \mathrm{~Hz}$ have large $\gamma \mathrm{s}$ whereas $218 \mathrm{~Hz}$ and $738 \mathrm{~Hz}$ or 861 and $981 \mathrm{~Hz}$ have smaller $\gamma \mathrm{s}$.

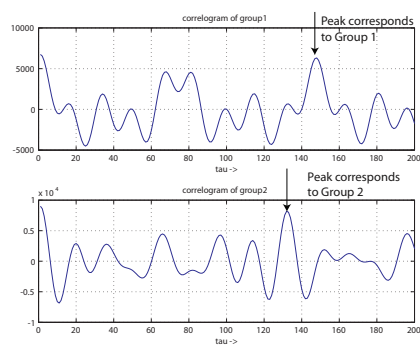

Fig. 6. Summary Autocorrelation for groups 1 and 2

pitch shift, pitch ambiguity, phase-invariance, pitch circularity, and the dominance region for pitch.," J. Neurophysiology, vol. 76, no. 3, pp. 1698-1734, 1996.

[4] R. Meddis and M. Hewitt, "Modeling the identification of concurrent vowels with different fundamental frequencies," J Acoust Soc Am, vol. 91, no. 1, pp. 233 245, 1992.

[5] J. F. Culling and C. J. Darwin, "Perceptual separation of simultaneous vowels: within and across-formant grouping by f0," JASA, vol. 93, no. 6, pp. 3454-67, 1993.

[6] B. Delgutte and N.Y.S. Kiang, "Speech coding in the auditory nerve: I. vowel-like sounds," J Acoust Soc Am, vol. 75, pp. 866-878, 1984.

[7] E. J. Baghdady, “Theory of stronger-signal capture in fm reception," in Proceedings of Institute of Radio Engineers, Aalborg, Denmark, April 1958, pp. 728-738.

[8] J. P. Costas, "Residual signal analysis," Proceedings of the IEEE, vol. 68, pp. 1351-1352, Oct. 1980.

[9] John P. Boyd, Chebyshev and Fourier Spectral Methods, Dover Publications, Inc., 31 East 2nd Street, Mineola, New York 11501, 2000.

[10] R. Patterson J. Holdsworth, I. Nimmo-Smith and P. Rice, "Implementing a gammatone filter bank," $M R C$ Applied Psychology Unit Tech. Rep, Feb 1988. 